\title{
COMPUTATION OF THE INTERIOR AND NEAR-FIELD FLOW OF A 2-kW CLASS HALL THRUSTER
}

\author{
Justin W. Koo ${ }^{*}$ and Iain D. Boyd ${ }^{\dagger}$ \\ Nonequilibrium Gas and Plasmadynamics Group \\ Department of Aerospace Engineering \\ University of Michigan \\ Ann Arbor, Michigan 48109 \\ James M. Haas ${ }^{\ddagger}$ and Alec D. Gallimore ${ }^{\S}$ \\ Plasmadynamics and Electric Propulsion Laboratory \\ Department of Aerospace Engineering \\ University of Michigan \\ Ann Arbor, Michigan 48109
}

\begin{abstract}
A time-accurate 2D axisymmetric Hybrid PIC-MCC model is used to simulate the plasma flow in the acceleration channel and near-field plume of a Hall thruster. The model is applied to the P5 Hall thruster due to known magnetic configuration and the availability of experimental data on the plasma properties in the interior and near-field plume for this thruster. Results for thruster performance and plasma properties are compared with this experimental data. In qualitative agreement with the measured data, the computations show significant ion acceleration in the plume near-field. Quantitative differences between the data sets suggest that fundamental $1 \mathrm{D}$ assumptions of the computational model regarding the electron energy equation begin to break down as the computational domain extends into the near-field plume.
\end{abstract}

\section{Introduction}

Computational models of the plasma properties inside Hall Thrusters have been developed by Komurasaki and Arakawa $^{1}$, Fife ${ }^{2}$, and Boeuf and Garrigues ${ }^{3}$. Due to the great sensitivity of these codes to the magnetic field configurations, one of the primary stumbling blocks to the further development of these models has been the lack of detailed experimental data from a thruster with a known magnetic field. With the advent of a new internal Hall thruster diagnostic by $\mathrm{Haas}^{4}$ and its operation on the non-commercial P5 thruster, experimental data from inside a Hall thruster with a known magnetic field is now available. The benefits of having a validated and reliable interior Hall Thruster model are many. First, such a model would aid in the design of newer thrusters, especially in higher power thrusters that will eventually exceed the pumping

\footnotetext{
${ }^{*}$ Graduate Student. Department of Aerospace

Engineering, Student Member AIAA, kooj@engin.umich.edu.

${ }^{\dagger}$ Associate Professor. Department of Aerospace Engineering. Senior Member AIAA.

${ }^{\ddagger}$ Research Scientist, AFRL, Edwards Air Force Base.

$\&$ Associate Professor. Department of Aerospace Engineering. Senior Member AIAA.
}

capabilities of present experimental facilities. Second, it would allow for accurate modeling of wall erosion, the primarly failure mode of these devices, saving enormous amounts of time and money presently devoted to experimental lifetime tests. Finally, a validated and reliable thruster model would provide much better input data for existing plume codes and thus improve the ability to assess spacecraft integration concerns regarding the sensitivity of solar panels and other electron equipment to impacts by high energy ions. These benefits motivated the development of a 2D axisymmetric Hybrid PIC-MCC code by Garrigues ${ }^{5}$ for the acceleration channel of the SPT- 100 Hall thruster.

Experimental results for the P5 thruster showed that a significant amount of the total potential drop occurs outside the Hall Thruster acceleration channel. Since electrostatic acceleration is the primary mechanism for ion acceleration, we hoped to first improve the computational model of Garrigues ${ }^{5}$ by extending the model to the larger geometry presented in this paper.

The following paper consists of first a description of the computational model, then a description of the experimental setup used to gather validation data, and finally a presentation of the results.

Copyright (C) 2001 by Justin Koo. Published by the American Institute of Aeronautics and Astronautics, Inc. with permission. 


\section{Computational Model}

\subsection{Overview}

The code presented in this paper is a time-accurate $2 \mathrm{D}$, azimuthally symmetric Hybrid PIC-MCC model of the plasma in the P5 acceleration channel and near-field. It is based on a quasineutral plasma description with a 1D electron energy model. Neutral $\mathrm{Xe}$ and $\mathrm{Xe}^{+}$ions are treated with a PIC-MCC model. Finally, the electrostatic potential is calculated using current continuity.

\section{$\underline{2.2 \text { Grids }}$}

This paper presents a computational Hall thruster model applied to a geometry that includes both the acceleration channel $(\mathrm{AC})$ and a portion of the nearfield plume. The $\mathrm{AC}$ geometry consists of the interior of the Hall thruster and covers a physical domain of 6.1 $\mathrm{cm}<\mathrm{r}<8.6 \mathrm{~cm}$ radially and $0.0 \mathrm{~cm}<\mathrm{z}<3.8 \mathrm{~cm}$ longitudinally, where $r$ is the radial location measured from the thruster centerline and $\mathrm{z}$ is the axial location measured from the anode. The near-field region covers a physical domain of $1.1 \mathrm{~cm}<\mathrm{r}<13.6 \mathrm{~cm}$ and $3.8 \mathrm{~cm}<$ $\mathrm{z}<10.0 \mathrm{~cm}$. Beginning the simulation at the thruster centerline $(\mathrm{r}=0 \mathrm{~cm})$ would have been preferable; however, magnetic field data was available only for the actual domain used. A 40x50 (r-z) cell rectangular grid is created to cover the entire region of $1.1 \mathrm{~cm}<\mathrm{r}<13.6$ $\mathrm{cm}$ and $0.0 \mathrm{~cm}<\mathrm{z}<10.0 \mathrm{~cm}$; however, a number of cells covering the actual thruster body were not used, so a total of only 1392 cells are actually used in the simulation.

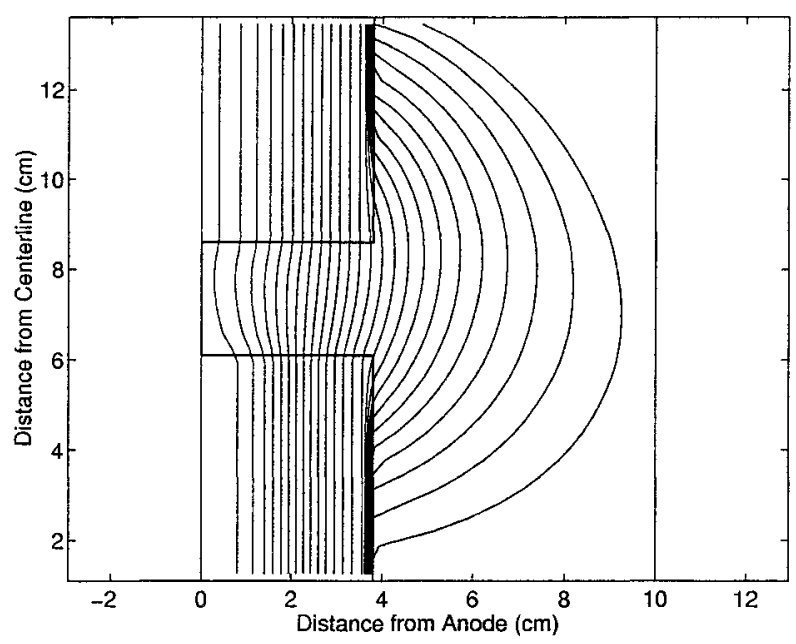

Figure 1. Magnetic field lines for P5 thruster

The magnetic field configuration of the P5, along with the large Hall current, causes the diffusion of electrons along magnetic field lines to be very large compared to diffusion across (perpendicular to) field lines.
Consequently, electrons are assumed to be isothermal along magnetic field lines, which allows the electron energy to be considered as a 1D problem with variation only perpendicular to magnetic field lines. In addition, this assumption allows for the idea of a thermalized potential which greatly simplifies the calculation of the electrostatic potential. For this reason, a second grid representing the magnetic field lines is necessary for the computational model. Since Hall thrusters with a power less than $5 \mathrm{~kW}$ are conventionally assumed to produce little or no magnetic self-field, the vacuum magnetic field measurements in Sec. 3.4 are sufficient to define the magnetic field grid. The magnetic field lines are represented by contours of the magnetic streamfunction defined as:

$$
\frac{\partial \lambda}{\partial z}=r B_{r} \frac{\partial \lambda}{\partial r}=-r B_{z}
$$

The resulting magnetic field grid is shown in Fig. 1.

\subsection{Heavy Particles}

\subsubsection{Neutrals}

Neutral Xe is modeled with a particle-in-cell (PIC) approach. Neutral macroparticles are injected at the anode every timestep with a half-Maxwellian velocity distribution characterized by a temperature of $1000 \mathrm{~K}$. The weighting of these macroparticles is varied to ensure that the total number of neutral macroparticles in the computational domain remains fairly constant (usually at around 40,000 ).

Neutral macroparticles are assumed to be collisionless and simply advect throughout the domain until they either hit a wall, leave the computational domain, or are ionized. If the neutral macroparticle encounters a wall, it is isotropically reflected with a half-Maxwellian velocity distribution based on a temperature of $1000 \mathrm{~K}$. Neutral macroparticles which exit the computational domain are no longer tracked and are assumed to have left the solution permanently. Neutral macroparticles lost to ionization are modeled with a Monte Carlo Collision (MCC) model. In this MCC model, a probability for ionization in each cell is constructed as follows:

$$
P=n_{e} k_{i}(\varepsilon) \Delta t
$$

where $n_{e}$ is the local electron density, $k_{i}(\varepsilon)$ is the ionization rate based on the electron energy, and $\Delta t$ is the timestep. A random number is then assigned to each neutral macroparticle in the cell and if the random number is less than or equal to the probability of ionization, that neutral macroparticle is destroyed. Averaged over many timesteps, this algorithm provides an accurate and simple mechanism to describe neutral 
depletion due to ionization. Neutral creation due to ion wall recombination is covered later in this section.

\subsubsection{Ions}

In this model, only singly charged xenon ions $\left(\mathrm{Xe}^{+}\right)$are considered. The $\mathrm{Xe}^{+}$macroparticles are created via a source term based on the local electron density $\left(n_{e}\right)$, the local neutral density $\left(n_{a}\right)$, and the ionization rate based on the electron energy $\left(\mathrm{k}_{\mathrm{i}}(\varepsilon)\right)$ via the following formula:

$$
S=n_{e} n_{a} k_{i}(\varepsilon)\left[\# / \mathrm{cm}^{3} / s\right]
$$

The new Xe+ macroparticles are created with a velocity based on a Maxwellian distribution with a temperature of $1000 \mathrm{~K}$.

At each timestep, this procedure is used to govern the number of new $\mathrm{Xe}^{+}$macroparticles created in each cell. If the number of $\mathrm{Xe}^{+}$macroparticles in the solution becomes too high, creation of new $\mathrm{Xe}^{+}$macroparticles is suspended until a sufficient number of old macroparticles have exited the computational domain.

Since the Larmor radius of $\mathrm{Xe}^{+}$particles is sufficiently large compared to the size of the Hall thruster $\mathrm{AC}(\mathrm{O}(1$ $\mathrm{m})$ vs $\mathrm{O}(1 \mathrm{~cm}))$, the $\mathrm{Xe}^{+}$particles are considered to be unmagnetized. Thus $\mathrm{Xe}^{+}$macroparticles move under the influence of the electric field via the simple set of equations:

$$
\begin{aligned}
& \vec{x}_{n+1}=\vec{x}_{n}+\vec{v}_{n} \Delta t \\
& \vec{v}_{n+1}=\vec{v}_{n}+\frac{e \vec{E}}{m_{i}} \Delta t
\end{aligned}
$$

If the $\mathrm{Xe}^{+}$macroparticles leave the domain at the far end of the near-field $(z=10 \mathrm{~cm}$ for this case), they contribute to the performance data for the thruster. Their velocity and energy are used to calculate the thrust, specific impulse, power, and efficiency of the thruster. However, if the $\mathrm{Xe}+$ macroparticles exit through either the top or bottom plane $(\mathrm{z}=13.6 \mathrm{~cm}$ or $\mathrm{z}$ $=1.1 \mathrm{~cm})$, they make no contribution to the overall performance of the thruster. This assumption is supported by the analysis that follows in Sec. 4.1.

\subsubsection{Wall Collisions}

At each timestep, the number and weight of $\mathrm{Xe}+$ macroparticles striking the different wall surfaces is determined and those $\mathrm{Xe}^{+}$macroparticles are destroyed. Neutral macroparticle are then created on the appropriate wall surfaces to account for the $\mathrm{Xe}^{+}$ macroparticles which have recombined at the wall surface. These new neutral macroparticles have a halfMaxwellian velocity based on a temperature of $1000 \mathrm{~K}$.

\subsubsection{Particle-In-Cell (PIC) Shape Factors}

Both $\mathrm{Xe}^{+}$and neutral macroparticles are mapped to the nodes on the grid at each time step to obtain conventional averaged densities. In the $z$-direction this is accomplished via simple linear interpolation. In the r-direction the shape factors used to map the macroparticle weights to the grid nodes are chosen to conserve charge density. The actual formulae used are from Ruyten ${ }^{6}$ :

$$
\begin{aligned}
& S_{n}=\frac{\left(r_{n+1}-r\right)\left(2 r_{n+1}+3 r_{n}-r\right)}{2\left(r_{n+1}^{2}-r_{n}^{2}\right)} \\
& S_{n+1}=\frac{\left(r-r_{n}\right)\left(3 r_{n+1}+2 r_{n}-r\right)}{2\left(r_{n+1}^{2}-r_{n}^{2}\right)}
\end{aligned}
$$

\subsection{Electrons}

\subsubsection{Energy Equation}

Since the electrons are assumed to be isothermal along magnetic field lines, the electron energy can be evaluated with a one-dimensional form of the electron energy equation. This equation is derived from the third moment of the Boltzmann equation by ignoring the time derivative and replacing the integrated RHS with a tabulated energy loss term based on the electron energy at the previous time step. The resulting electron energy equation, shown below, has terms of energy flux and thermal flux on the left hand side and source terms for energy from the electric field and from collisions on the right hand side.

$$
\frac{\partial}{\partial z}\left[\frac{5}{3} n_{e} v_{e, \perp} \varepsilon_{e}-\frac{5}{3} n_{e} D_{e, \perp} \frac{\partial \varepsilon_{e}}{\partial z}\right]=-e n_{e} v_{e, \perp} E_{\perp}-n_{e} \varepsilon_{e} v\left(\varepsilon_{e}\right)
$$

In this equation, $\varepsilon_{\mathrm{e}}$ represents the mean electron energy, $v_{e, \perp}$ represents the electron velocity perpendicular to the field lines, $D_{e, \perp}$ represents the thermal diffusivity perpendicular to the field lines, and $v\left(\varepsilon_{\mathrm{e}}\right)$ represents the total electron energy loss frequency which consists of an ionization energy loss rate and the following formula (which is meant in part to simulate the loss of high energy electrons to the wall):

$$
\nu\left(\varepsilon_{e}\right)_{\text {walls }}=0.2 * 10^{7} \exp \left(-\frac{20}{\varepsilon_{e}}\right)[1 / s]
$$

When properly discretized, Eq. 6 can be formed into a tridiagonal system. To obtain the input values for the electron energy equation, the relevant quantities are averaged vertically along grid lines at various axial locations throughout the computational domain. There is some error inherent to this approach, especially in the 
near-field, which should instead average along the magnetic field lines. More discussion on this topic will follow in Sec. 4.4. With the boundary conditions of zero-slope on the anode side and an imposed electron energy at the exit plane, this system can be integrated at each time step. During the simulation, the electron energy is limited to $100 \mathrm{eV}$.

\subsection{Electric Field}

\subsubsection{Electron Mobility}

Classical electron mobility across magnetic field lines is:

$$
\mu_{e, \perp}=\frac{q_{e}}{m v_{m}} \frac{1}{1+\frac{\omega_{c, e}{ }^{2}}{v_{m}{ }^{2}}}
$$

Where $v_{m}$ is the electron momentum exchange collision frequency and $\omega_{i, \mathrm{e}}$ is the electron cyclotron frequency. The collision frequency is based primarily on electronneutral collisions, which are modeled by the following approximation,

$$
\left(v_{m}\right)_{\text {neutrals }}=2.5 * 10^{-7} n_{a}[1 / \mathrm{s}]
$$

where $n_{a}$ is neutral density measured in particles $/ \mathrm{cm}^{3}$. In order to account for the increased electron mobility in the $\mathrm{AC}$, the electron mobility across magnetic field lines can be modified by adding a term to the collision frequency representing an electron-wall collision term. As in the work of Boeuf and Garrigues ${ }^{3}$, this electronwall collision term is chosen to be:

$$
\left(v_{m}\right)_{\text {walls }}=0.2 * 10^{7}[1 / s]
$$

The result is the total electron momentum exchange collision frequency used in the $\mathrm{AC}$ region of the computational model:

$$
\left(v_{m}\right)_{\text {total }}=\left(v_{m}\right)_{\text {neutrals }}+\left(v_{m}\right)_{\text {walls }}
$$

\subsubsection{Thermalized Potential}

In order to cast the electron momentum equation in a 1D form, it is necessary to use the idea of the "thermalized potential," an approach pioneered by Morozov $^{7}$ and implemented by Fife ${ }^{2}$. Due to the presence of the Hall current, it is assumed that the electrons reach an equilibrium between the pressure force and the electric force along magnetic field lines. This can be represented by the following equation where $k_{B}$ represents the Boltzmann constant and $t_{\|}$ represents the unit vector along the magnetic field line.

$$
\frac{\partial\left(n_{e} k_{B} T_{e}\right)}{\partial t_{\|}}=e n_{e} \frac{\partial V}{\partial t_{\|}}
$$

Integrating this equation with the assumption that the electron temperature is constant along magnetic field lines leads to:

$$
V-\frac{k_{B} T_{e}}{e} \ln \left(\frac{n_{e}}{n_{e, 0}}\right)=V^{*}(\lambda)
$$

where $V^{*}(\lambda)$ is called the thermalized potential and represents a quantity that is conserved along magnetic field lines (lines of constant $\lambda$ ). (In this model, $\mathrm{n}_{\mathrm{e}, 0}$ is assumed to be the electron density at the middle of the exit plane.) The grids of equal $\lambda$ spacing used in these computations are shown in Sec. 2.2.

\subsubsection{Electron Momentum}

Using the same approach as Boeuf and Garrigues ${ }^{3}$ and $\mathrm{Fife}^{2}$, the electron momentum can be written as:

$$
J_{e, \perp}=-e n_{e} \mu_{e, \perp} r B \frac{\partial V^{*}}{\partial \lambda}
$$

This formula assumes a constant relation between the perpendicular diffusion and mobility coefficients which is set by the mean value of the electron temperature in the region within $5 \mathrm{~mm}$ of the anode.

\subsubsection{Current Conservation}

Total current is conserved through the computational domain in the radial direction (with the exception of small amounts of current leaking out the top of the solution). The total current can be written as the sum of the electron and ion currents through any single magnetic field line as shown below:

$$
I_{T}=-\int 2 \pi e n_{e} \mu_{e, \perp} r^{2} B \frac{\partial V^{*}}{\partial \lambda} d l+\int 2 \pi e n_{i} u_{i, \perp} r d l
$$

(In this formula, the integration, $\mathrm{dl}$, is along any single magnetic field line.) Since the derivative with respect to lambda is constant along the field line, it can be brought out of the integral and this formula can be rewritten as:

$$
\frac{\partial V^{*}}{\partial \lambda}=\frac{I_{T}-\int 2 \pi e n_{i} u_{i, \perp} r d l}{-\int 2 \pi e n_{e} \mu_{e, \perp} r^{2} B d l}=-E(\lambda)
$$

Finally, since the total voltage drop between the anode and exit plane is known, so is the corresponding thermalized potential drop, as shown below:

$$
V_{\max }^{*}=\int_{\lambda(\text { anode })}^{\lambda(\text { exitplane })} E(\lambda) d \lambda V_{\max }^{*}
$$

Substituting in Eq. 16 leads to the following formulation for the thermalized potential drop: 


$$
V_{\max }^{*}=\int_{\lambda(\text { anode })}^{\lambda(\text { exitplane) }} \frac{I_{T}-\int 2 \pi e n_{i} u_{i, \perp} r d l}{\int 2 \pi e n_{e} \mu_{e, \perp} r^{2} B d l} d \lambda
$$

Since $\mathrm{I}_{\mathrm{T}}$ is constant throughout the domain, it can be brought out of the integral and the current conservation equation can finally be written as:

$$
I_{T}=\frac{V_{\max }^{*}+\int_{\lambda(\text { anode })}^{\lambda(\text { (exitplane) }} \frac{\int 2 \pi e n_{i} u_{i, \perp} r d l}{\int 2 \pi e n_{e} \mu_{e, \perp} r^{2} B d l} d \lambda}{\int_{\lambda(\text { anode })} \frac{1}{\int 2 \pi e n_{e} \mu_{e, \perp} r^{2} B d l} d \lambda}
$$

In this form it is clear that the total current is described entirely in terms of known variables. Once the total current is obtained, the electric field and thermalized potential on the lambda grid can be retrieved using Eq. 16 and Eq. 17. The thermalized potential is then expanded along the field lines to the $2 \mathrm{D}$ domain and the appropriate density correction is used to retrieve the electrostatic potential. Poisson's equation is then used to derive the electric field throughout the $2 \mathrm{D}$ domain.

\subsection{Computational Details}

\subsubsection{Code Execution}

The computational model is compiled with FORTRAN 77 to run on a Sun Ultra $10 / 440 \mathrm{MHz}$ workstation. A simulation typically contains 40,000 ion macroparticles and 30,000 neutral macroparticles. The minimum timestep for a PIC simulation is typically chosen to ensure that the fastest moving particles in the simulation will remain within the smallest computational cell for at least one timestep. In this code, that requirement is strictly enforced by limiting the timestep to one-fifth of the cell travel time for a perfectly accelerated ion. This leads to a timestep of $1.98 \times 10^{-8}$ seconds. A total of $200,000-400,000$ timesteps are typically used to drive the simulations to a "steady-state" regime where the performance parameters remain constant (with the usual plasma oscillation). This takes on the order of 10-15 hours.

\section{Experimental Setup}

\subsection{UM/AFRL P5 Hall Thruster}

The UM/AFRL P5 Hall thruster is a 5-kW class thruster designed and developed at the University of Michigan Plasmadynamics and Electric Propulsion Lab (PEPL) by Haas et $\mathrm{al}^{8}$. The experimental data presented in this paper are taken at lower power levels with a fixed potential drop between anode and cathode of $300 \mathrm{~V}$.
Performance parameters of this thruster will be presented later in this paper.

\subsection{Experimental Facility}

All experiments were conducted in the University of Michigan's $6 \mathrm{~m}$ diameter by $9 \mathrm{~m}$ long Large Vacuum Test Facility (LVTF). The pumping system consists of seven CVI model TM-1200 Re-Entrant Cryopumps providing a measured xenon pumping speed of 240,000 1/s. The ultimate base pressure of the facilityis less than $2 \times 10^{-7}$ Torr. Four of the seven cryopumps were used for these tests. The resulting operating pressures for this experiment were $5.5 \times 10^{-6}$ Torr and $9.6 \times 10^{-6}$ Torr when corrected for Xenon and corresponded to discharge currents of 5.4 A and 10.0 A, respectively.

\subsection{HARP}

The emissive and double Langmuir probe data were obtained inside, and in the near-field region of, the Hall thruster discharge chamber using the PEPL HARP (High-speed Axial Reciprocating Probe) system. The HARP system allows the probe to be inserted into, and removed from, the thruster in less than $100 \mathrm{~ms}$. This short residence time enables measurements to be made with very little perturbation to thruster operation. The extent of thruster perturbation is determined by monitoring the discharge current during probe movement. Use of the emissive and Langmuir probes caused a slight perturbation in the discharge current but this remained less than $10 \%$ of the nominal discharge current value during all measurements. Further details are found in Ref. 10.

\subsection{Magnetic Field Configuration}

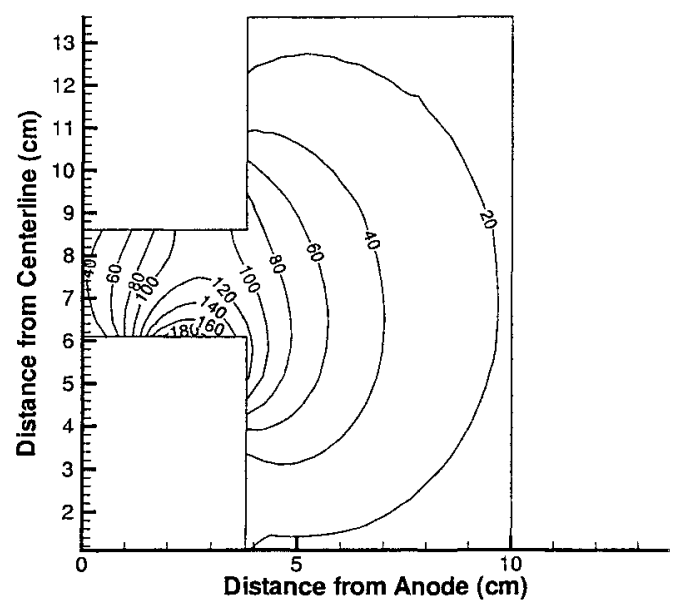

Figure 2. Radial magnetic field (Gauss) 
The experimental data presented in this paper were taken at a single magnetic field configuration. This field was measured in vacuum by Martinez et $\mathrm{al}^{9}$. The maximum magnitude of the magnetic field was $202 \mathrm{G}$ next to the inner wall about halfway down the AC. A corresponding peak in the radial magnetic field of 195 $\mathrm{G}$ is observed in roughly the same spatial location. This asymmetry is largely the result of a higher current setting in the inner electromagnets than the outer electromagnets of the P5 for this operating mode. The radial magnetic field plot is shown in Fig. 2.

\subsection{Results and Discussion}

\subsection{Performance Parameters}

The experimental data presented here are obtained by holding the discharge voltage at $300 \mathrm{~V}$. At the $1.6 \mathrm{~kW}$ power level, the anode mass flow rate of Xe was 6.2 $\mathrm{mg} / \mathrm{s}$ and the discharge current was $5.4 \mathrm{~A}$ and at the 3 $\mathrm{kW}$ power level, the anode mass flow rate of Xe was $10.7 \mathrm{mg} / \mathrm{s}$ and the discharge current was $10 \mathrm{~A}^{10}$

Due to the assumption of $25 \mathrm{~V}$ needed by the external cathode for electron emission, the computational results are obtained with an imposed voltage drop of $275 \mathrm{~V}$ between the anode and near-field boundary of the plume. Anode mass flow rates of $7,9,11$, and $13 \mathrm{mg} / \mathrm{s}$ are tested.

Xe Mass Flow Rate (mg/s) Current (A) Power (W)

$\begin{array}{lrr}\text { Experimental }-6.2 & 5.4 & 1.60 \\ \text { Computational }-7.0 & 4.2 & 1.15 \\ \text { Experimental }-10.7 & 10.0 & 3.00 \\ \text { Computational }-11.0 & 7.6 & 2.09\end{array}$

Xe Mass Flow Rate (mg/s) Thrust (mN) Isp (s)

$\begin{array}{ccr}\text { Experimental }-6.2 & 95.0 & 1550 \\ \text { Computational }-7.0 & 46.6 & 678 \\ \text { Experimental }-10.7 & 175.0 & 1670 \\ \text { Computational }-11.0 & 96.1 & 890\end{array}$

Table 1. Thruster Performance

As can be seen from the results in Table 1, the computational data follow the same trends as the experimental data but consistently under-predicted the measured thruster performance by about $50 \%$.

Note that the computational data presented here is timeaveraged data taken from a simulation with an anode Xe flow rate of $11 \mathrm{mg} / \mathrm{s}$ and the experimental data presented here is taken from the $10.7 \mathrm{mg} / \mathrm{s}$ anode $\mathrm{Xe}$ flow rate run.

\subsection{Plasma Potential}

The experimental results show the electrostatic potential following a gradual decline from the anode until within about a centimeter of the exit. Over the next $2 \mathrm{~cm}$, the potential drops sharply by about $175 \mathrm{~V}$. A significant amount of potential drop, between 75-100 $\mathrm{V}$, occurs in the near-field plume. Throughout the plume, a pronounced potential "jet" is visible. This behavior can be seen in Fig. 3 .

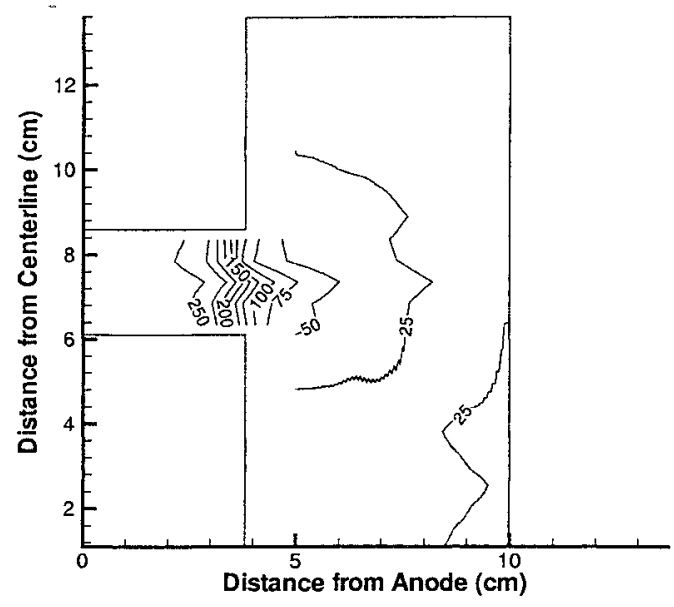

Figure 3. Measured plasma potential (V)

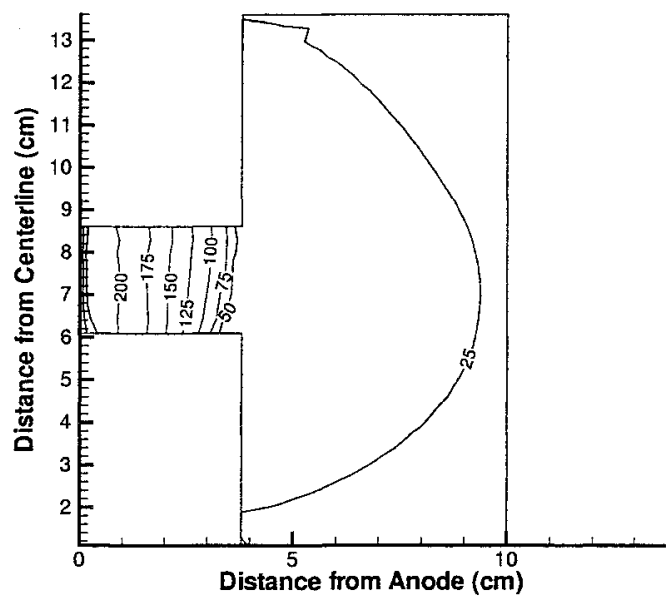

Figure 4. Computed plasma potential (V)

The corresponding computational results at $11 \mathrm{mg} / \mathrm{s}$ are shown above in Fig. 4 . Within $1 \mathrm{~cm}$ of the anode, the computational model has already given up $75 \mathrm{~V}$ of the plasma potential. The gradient down to the exit plane value of $40 \mathrm{~V}$ is uniform through the AC. Finally, a large region of fairly significant plasma potential extends over 5 centimeters of the near-field plume. 


\subsection{Plasma Density}

The experimental results obtained show a thin ionization region at $\mathrm{z}=3.3 \mathrm{~cm}$ with a peak number density of $2.5 \times 10^{12}$ particles $/ \mathrm{cm}^{3}$ centered within a larger zone beginning at $\mathrm{z}=3.0 \mathrm{~cm}$ with an average number density of around $1 \times 10^{12}$ particle $/ \mathrm{cm}^{3}$. This is displayed below in Fig. 5.

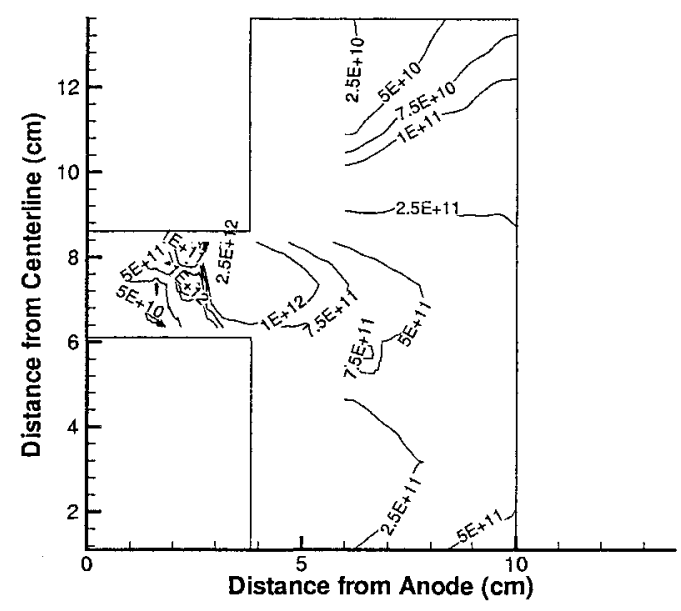

Figure 5. Measured plasma density $\left(\# / \mathrm{cm}^{3}\right)$

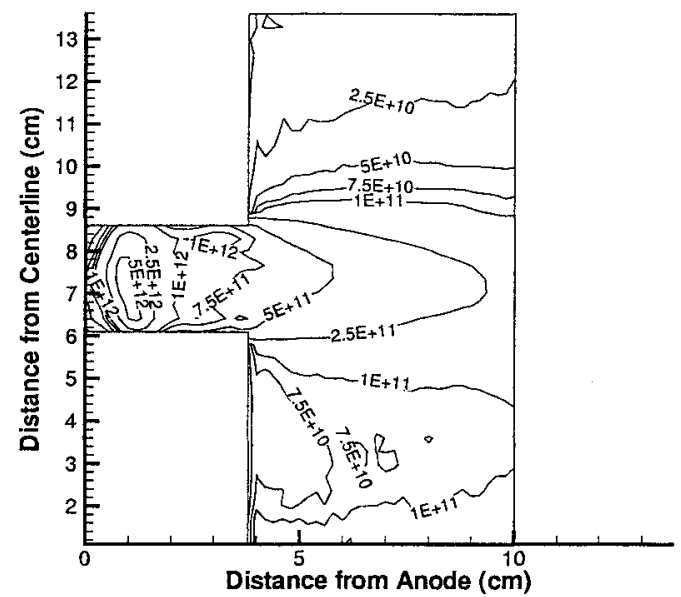

Figure 6. Computed plasma density $\left(\# / \mathrm{cm}^{3}\right)$

By comparison, the computational results, presented above in Fig. 6, at the same mass flow rate show a large region of $\mathrm{Xe}^{+}$developing at about $1 \mathrm{~cm}$ from the ariode. The peak ion density in this region is about $6 \times 10^{12}$ particles $/ \mathrm{cm}^{3}$. In the near-field, there is also a much higher ion density closer to the centerline of the thruster than at the outer edge. Of course, by the time the ions have reached the exit $(\mathrm{z}=10 \mathrm{~cm})$, they have both a high axial momentum and a high axial velocity so that the plasma density begins to drop.

\subsection{Electron Energy}

The experimental results obtained show a sharp spike in electron energy (of $30 \mathrm{eV}$ ) near the ionization zone at $\mathrm{z}$ $=3 \mathrm{~cm}$. The electron energy then drops rapidly to a very low background level of approximately $2-3 \mathrm{eV}$ throughout the near-field domain. These experimental results for electron energy can be seen in Figs. 7 and 8.

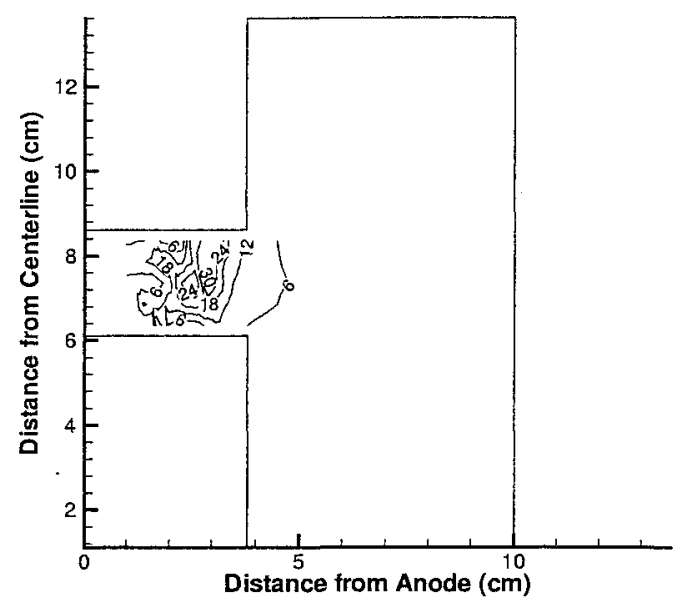

Figure 7. Measured electron energy (eV)

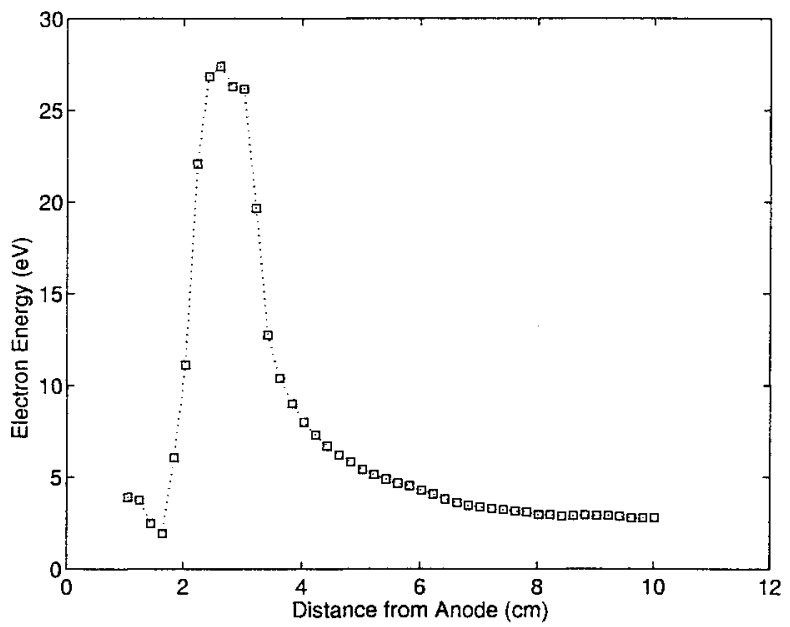

Figure 8. Measured AC centerline electron energy

The computational results obtained are strictly onedimensional due to the formulation of the electron energy equation. The near-field boundary condition on the model is that the electrons enter the simulation with an energy of $1 \mathrm{eV}$. The data shown in Fig. 9 represent the average electron energy along the centerline of the AC channel. 


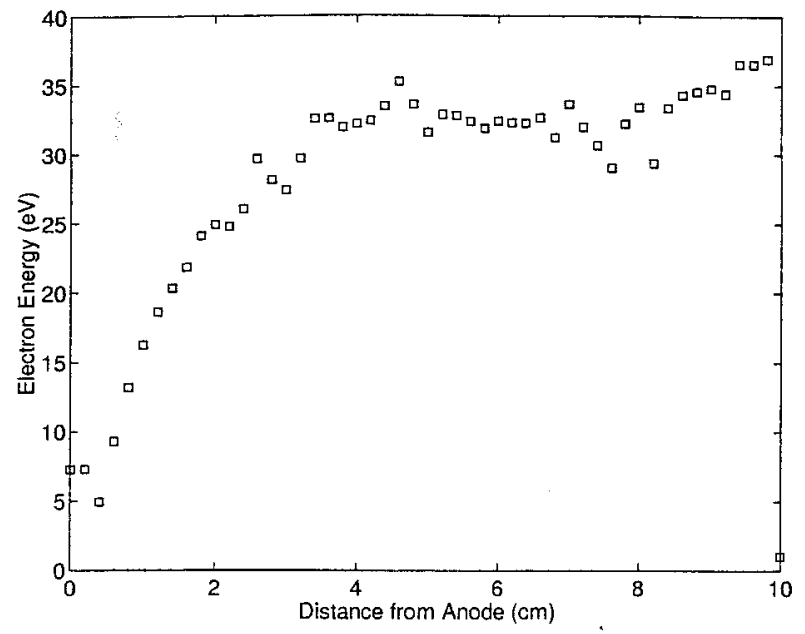

Figure 9. Computed electron energy

\subsection{Discussion}

There are a number of major discrepancies that are observed when comparing the experimental and computational results. Of these, the three of greatest concern are the underprediction of the gross performance parameters, the location and magnitude of the peak ionization zone in the $\mathrm{AC}$, and the high electron energy in the plume.

As observed in Sec. 4.1, the gross performance parameters of the computational model are up to $50 \%$ lower than the same performance parameters obtained experimentally. This behavior was first believed to be a consequence of allowing the plasma to escape through the upper and lower boundaries of the solution without contributing to the performance parameters of the solution. In making this assumption, it was assumed that during the span of $6.2 \mathrm{~cm}$ into the near-field, the plume would not diverge significantly. This assumption is tested by the following plot of averaged ion velocity for the $11 \mathrm{mg} / \mathrm{s}$ case in Fig. 10.

From this plot, it is clear that the high velocity jet formed as the ions exit the thruster undergoes a rapid expansion radially as it moves into the near-field region. Clearly, some fraction of high velocity ions $(-4,000 \mathrm{~m} / \mathrm{s})$ "leak" out both the top and bottom of the domain. However, upon detailed calculation, it was determined that the thrust contribution by particles crossing the top plane was about $1 \%$ and the contribution by particles crossing the bottom plane was about $0.05 \%$. Thus, the method used for performance calculation is not the cause for the underprediction of performance.

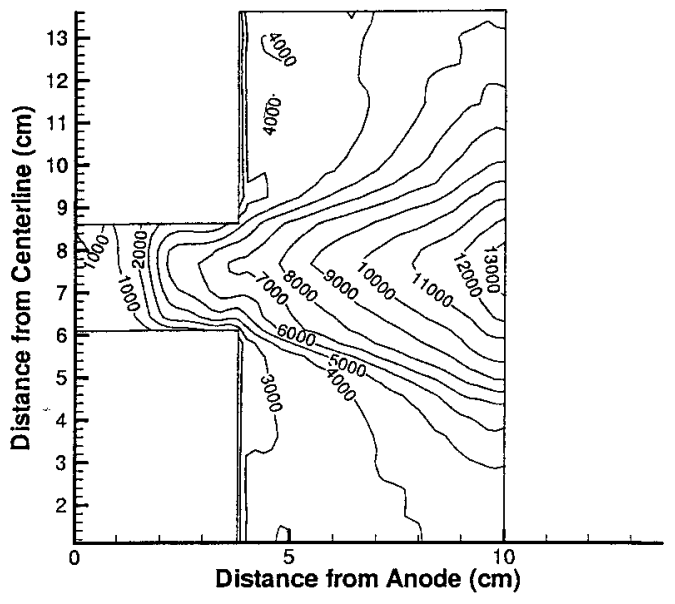

Figure 10. Computed ion velocity $(\mathrm{m} / \mathrm{s})$

Using the time-averaged ion data, the thrust at each axial location was evaluated throughout the domain. The results reveal that the thrust is increasing monotonically in the axial direction throughout the AC and plume. Evidently, the ions are still undergoing significant acceleration throughout the plume, as shown in Fig. 11.

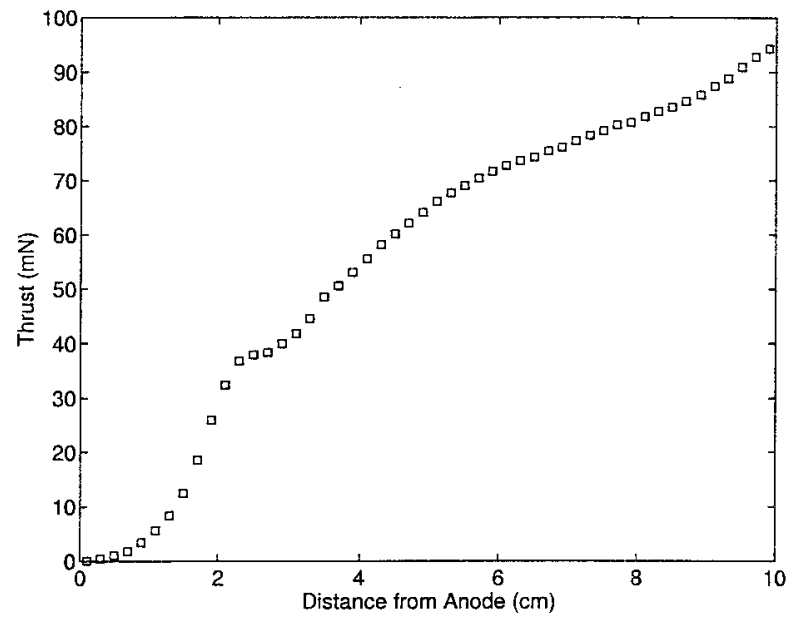

Figure 11. Computed thrust at various axial locations

The presence of a significant acceleration region for the P5 extending beyond $10 \mathrm{~cm}$ from the exit plane of the thruster was also observed experimentally by Williams ${ }^{11}$. Thus, extending the computational domain further into the near-field offers real hope in improving gross performance parameters.

The second major concern focused on the relative magnitude of the peak ionization zone in the computational result (over twice as high as the experimental result) and its extreme upstream position (within $1 \mathrm{~cm}$ of the anode). The explanation for the 
relatively higher $\mathrm{Xe}^{+}$densities is almost certainly based on the relatively higher electron energies present in this solution. With an average electron energy in the $\mathrm{AC}$ of $21.5 \mathrm{eV}$ for the simulation, the ionization rate coefficient $k_{i}(\varepsilon)$ is roughly twice as high as that for the $10-15 \mathrm{eV}$ average electron energy in the $\mathrm{AC}$ for the experimental data. The position of the main ionization zone in this model is best explained by the very high neutral densities near the anode, as shown in Fig. 12.

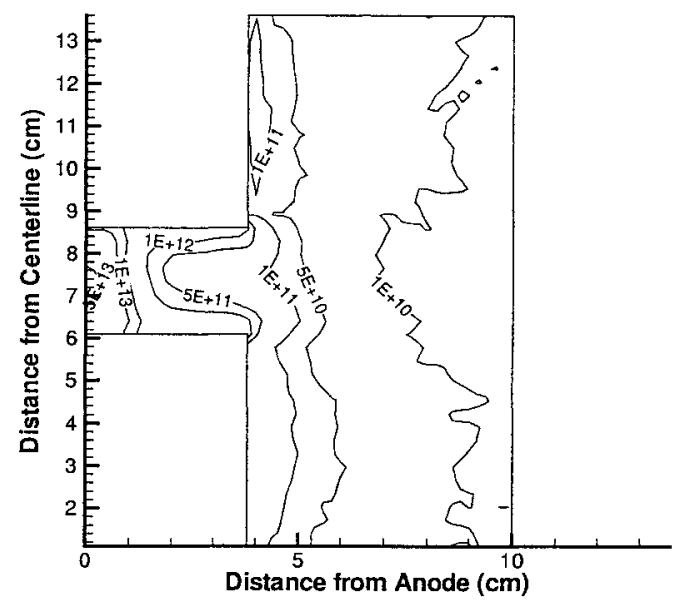

Figure 12. Computed neutral density $\left(\# / \mathrm{cm}^{3}\right)$

Although this plot does not provide a fundamental explanation for why the ionization zone is so far up the $\mathrm{AC}$, it does help rationalize the computational results obtained, namely, that the very high neutral density near the anode, plus the relatively high electron energy, is sufficient to cause a great deal of ionization within a short distance from injection.

Finally, the last major area of concern is that the average electron energy in the near-field plume is actually higher than the electron energy in the acceleration channel. From the experimental data, it is clear that the electron energy should asymptote to 2-3 $\mathrm{eV}$ as the distance from the thruster increases.

Since the computational results indicate that the neutral density and plasma density decreases rapidly as the solution is extended farther and farther into the nearfield, electron-neutral collision rates in this region should be far smaller than observed in the AC. In addition, the absence of wall collision in the near-field further minimizes the contribution to the electron energy loss of collision in the near-field.

One possible remedy for the plume electron energy might be, as mentioned in Sec. 2.4.1, a more physically accurate manner of evaluating the electron energy.
This approach would be to collect input data for the electron energy equation along magnetic field lines and to project the solution back to the $2 \mathrm{D}$ domain along magnetic field lines. The averaging of input quantities in the radial direction at constant $\mathrm{z}$, as implemented in this simulation, causes little error in the $\mathrm{AC}$ where the magnetic field lines are almost vertical, but does cause major problems in the near-field where the magnetic field lines are almost semicircular.

This solution will certainly be attempted in the near future; however, the possibility remains that a $1 D$ electron energy equation is not sufficient to describe the behavior of the electron energy seen in Fig. 7. Near the exit plane of the P5 Hall thruster, where the magnetic field lines are nearly vertical, the electron energy along some of the field lines varies all the way from 6 to 30 $\mathrm{eV}$. This indicates that the thermal flux (based on the gradient of electron energy) and collision terms (based on the magnitude of electron energy) can vary significantly along a single field line. Consequently, it is seems necessary to use a $2 \mathrm{D}$ electron energy equation to properly capture the effect of these spatial variations on the evolution of the electron energy.

The poor performance of the computational solution is partly due to the shape of the electric potential. The last $25 \mathrm{~V}$ of the potential drop accelerates the ions almost uniformly through a semicircle centered at $\mathrm{r}=7.3 \mathrm{~cm}$ and $\mathrm{z}=3.8 \mathrm{~cm}$. This manages not only to divert ions away from the exit plane, but also to deprive them of the chance to gain additional axial momentum.

Certainly the simplest explanation for the shape of the equipotentials is the use of a $1 \mathrm{D}$ current conservation model to calculate the potential. In the near-field, the plasma density is not large, so the thermalized potential and electrostatic potential are relatively similar. Thus, when the updated thermalized potential is "smeared" out along the magnetic field lines at each iteration, so is the electrostatic potential.

\section{$\underline{5.0 \text { Conclusions }}$}

A time-accurate $2 \mathrm{D}$ computational model was presented for the acceleration channel and near-field plume of the P5 Hall thruster. Comparison of the computational results with experimental data provided many interesting observations about the computational model. First, it produced the correct trends versus anode mass flow rate in thrust, specific impulse, and discharge current, although it consistently underpredicted these characteristics by up to $50 \%$. Second, the model correctly predicted a great deal of ion acceleration outside the acceleration channel. By extending the near-field domain even further into the plume, the 
performance predictions of the code should compare more favorably to experimental results. Finally, the existing 1D electron energy equation formulation used in the model proved to be incapable of correctly modeling the electron energy in the near-field plume of the thruster.

\subsection{Future Work}

A number of straightforward changes to the code should now be made. The computational domain should be extended in the radial direction to the thruster centerline and to at least double its present dimension in the axial direction. This should provide an immediate boost to the overall thruster performance in the code.

As this computational model become more developed, a comprehensive treatment of the wall boundary conditions will be desirable. There are significant wall boundary effects, such as electron energy loss due to wall collisions and electron mobility changes due to secondary electron emission, that are presently modeled in a somewhat ad hoc manner. Developing more physically motivated models for these electron-wall interactions should result in a more robust and accurate code.

Of course, the electron energy should be changed to actually produce isothermal electrons along magnetic field lines. However, the fundamental question must be addressed as to whether the existing $1 \mathrm{D}$ electron energy equation formulation can be simply modified, or must be completely replaced by a 2D formulation in order to bring the computed electron energy in the plume back in line with the 2-3 eV electron energies observed experimentally. The additional boundary conditions of a 2D formulation would provide a useful way to bound the plume energy spatially. The bottom boundary condition with an expanded domain would be as an axis of symmetry, the top and extreme right near-field boundaries would be set to $2-3 \mathrm{eV}$, and adiabatic wall conditions would be applied to the rest of the boundaries.

\section{Acknowledgement}

The first two authors gratefully acknowledge financial support from the TRW Foundation and from the Department of Energy through a Computational Science Graduate Fellowship

\section{$\underline{\text { References }}$}

${ }^{1}$ Komurasaki, K. and Arakawa, Y., "Two-Dimensional Numerical Model of a Plasma Flow in a Hall Thruster,"
Journal of Propulsion and Power, Vol. 11, 1995, pp. 1317-1323.

${ }^{2}$ Fife, J. M., "Hybrid-PIC Modeling and Electrostatic Probe Survey of Hall Thrusters," Doctoral Thesis, Massachusetts Institute of Technology, Department of Aeronautics and Astronautics, September 1998.

${ }^{3}$ Boeuf, J.-P. and Garrigues, L., "Low Frequency Oscillations In a Stationary Plasma Thruster," Journal of Applied Physics, Vol. 84, 1998, pp. 3541-3544.

${ }^{4}$ Haàs, J.M., Gallimore, A.D., "An Investigation of Internal Ion Number Density and Electron Temperature Profiles in a Laboratory-Model Hall Thruster," AIAA00-3422, $36^{\text {th }}$ Joint Propulsion Conference, Huntsville, AL, July 16-19, 2000.

${ }^{5}$ Boyd, I.D. Garrigues, L., Koo, J., and Keidar, M., "Progress in Development of a combined Device/Plume Model for Hall Thrusters," AIAA-00-3520, $36^{\text {th }}$ Joint Propulsion Conference, Huntsville, AL, July 16-19, 2000.

${ }^{6}$ Ruyten, W.M., "Density-Conserving Shape Factors for Particle Simulations in Cylindrical and Spherical Coordinates," Journal of Computational Physics, Vol. 105, 1993, p. 228.

${ }^{7}$ Morozov, A. I., Esipchuk, Yu. V., Tilinin, G. N., Trofimov, A. V., Sharov, Yu. A., Shchepkin, G. Ya., "Plasma Accelerator With Closed Electron Drift and Extended Acceleration Zone," Soviet Journal of Plasma Physics, Vol. 17, 1972, p.38.

${ }^{8}$ Haas, J.M., Gulczinski, F.S., Gallimore, A.D., Spanjers, G.G., Spores, R.A., "Performance Characteristics of a $5 \mathrm{~kW}$ Laboratory Hall Thruster," AIAA-98-3503, 34 $4^{\text {th }}$ Joint Propulsion Conference, Cleveland, OH, July 12-15, 1998.

${ }^{9}$ Martinez, R. A., Hofer R.R., Private Communication.

${ }^{10}$ Haas, J. M., "Low-Perturbation Interrogation of the Internal and Near-Field Plasma Structure of a Hall Thruster Using a High Speed Probe Position System," Doctoral Thesis, University of Michigan, Department of Aerospace Engineering, February 2001.

${ }^{11}$ Williams, G. J. Jr., Smith, T. B., Gulczinski, F. S., Beal, B. E., Gallimore, A. D., Drake, R. P., "Laser Induced Fluorescence Measurement of Ion Velocities in the Plume of a Hall Effect Thruster," AIAA-99-2424, $35^{\text {th }}$ Joint Propulsion Conference, Los Angeles, CA, June 20-23, 1999. 